\title{
An investigation of green iridescence on the mollusc Patella granatina
}

\author{
D J Brink and N G van der Berg \\ Department of Physics, University of Pretoria, Pretoria 0002, South Africa \\ Received 29 September 2004, in final form 4 November 2004 \\ Published 6 January 2005 \\ Online at stacks.iop.org/JPhysD/38/338
}

\begin{abstract}
In this paper we investigate the relatively rare phenomenon of iridescence on the outer surface of seashells (not the well known pearly inner surfaces). Using reflection spectroscopy and scanning electron microscopy we show that rows of iridescent green spots on the mollusc Patella granatina are caused by a thin-film stack buried about $100 \mu \mathrm{m}$ below the rough outer surface of the shell. The high-density layers in the stack seem to be made of crystalline aragonite, but according to Raman spectroscopy and ellipsometry measurements the low-density layers as well as the bulk of the shell wall are a mixture of porous aragonite and organic materials such as carotenoids.
\end{abstract}

\section{Introduction}

A wide variety of biological species exhibit bright iridescent colours created by interference or diffraction from microstructures made from essentially colourless materials [1-5]. However, as pointed out in an earlier paper [6], these structural colours are relatively rare for seashells (we are not referring to the pearly appearance on the inside of most shells, but to the outer surface).

In this paper we study one of the few exceptions: the mollusc Patella granatina (commonly known as the granite limpet) found along the west coast of southern Africa. This shell has an appearance very different from that of the limpet Helcion pruinosus investigated earlier [6] in that it belongs to a different genus and species and its outer surface has a rough wavy structure (see figure 1) instead of the smooth rounded surface of Helcion pruinosus. Numerous rows of rectangular bright green iridescent spots run from the apex of the shell to the perimeter and in an even more pronounced way than for the previous species. The iridescence is only visible when the shell is under water and viewed at close to grazing incidence from the apex to the perimeter.

In the work presented here it is our main aim to thoroughly investigate the optical characteristics of the green iridescence in Patella granatina by combining various optical techniques with electron microscopy. We employed more precise techniques (ellipsometry and Raman spectroscopy) and a more sophisticated method of interpreting the reflectance spectra than before. The results are compared with those of the earlier investigation of Helcion pruinosus.

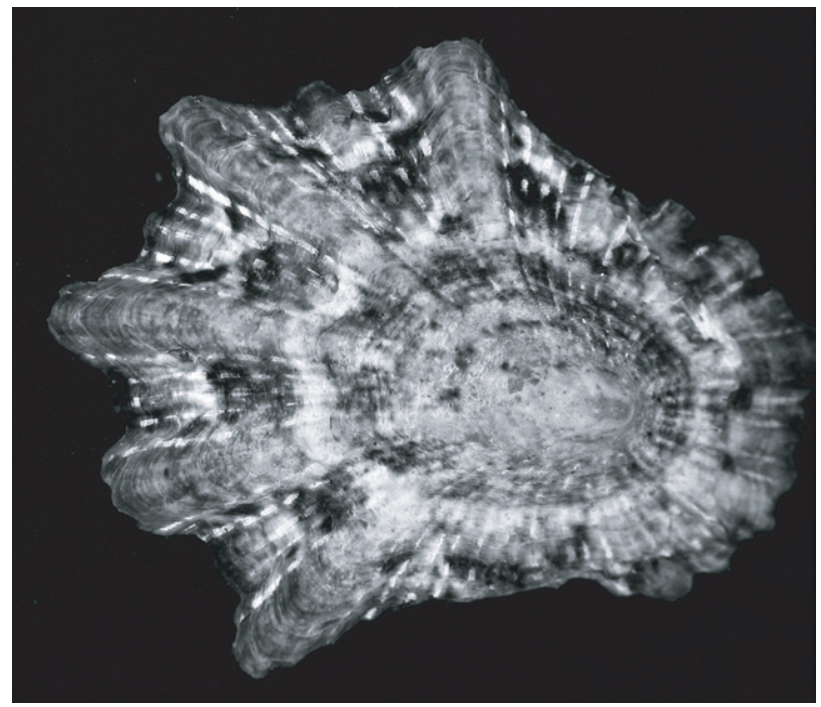

Figure 1. The top view of Patella granatina. The lighter rectangular spots organized in radial rows appear iridescent when viewed at near grazing incidence from the apex to the perimeter. Due to excessive light scattering from the rough corroded outer surface the iridescence is only visible when the shell is wet or immersed in water.

\section{Experimental details}

Live specimens were collected from shallow pools near the Langebaan estuary north of Cape Town. As pointed out above, no colours are visible on the dry shells due to scattering from the rough corroded outer layer. In order to observe and quantify 


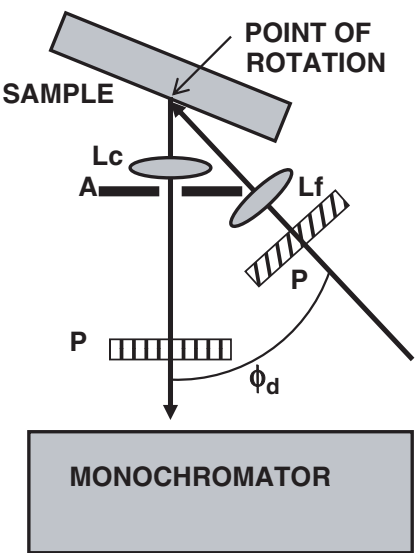

Figure 2. Experimental layout for reflectance measurements. A collimated beam of white light is polarized by a linear polarizer (P) and focused onto the sample using a lens (Lf). Light scattered from the sample is collected by lens (Lc). The scattering angle is limited by a slit aperture (A), and a second polarizer (P) removes any depolarized light before entering the monochromator. The deviation angle $\left(\phi_{\mathrm{d}}\right)$ is the angle between the input and output beams. The sample can be rotated in the plane of the paper about a point at the intercept of the input and output beams.

reflected light it was essential to polish the surface with a $6 \mu \mathrm{m}$ diamond paste on a revolving pad. We took special care to keep the polished surface parallel to the plane of the original (local) surface and to remove the absolute minimum amount of material. Reflectance spectra were recorded from these samples using a $0.64 \mathrm{~m}$ Jobin Yvon monochromator and a cooled S20 photomultiplier.

As shown in figure 2, a collimated beam from a white tungsten-halogen lamp (1.5 mm beam diameter) was used to illuminate the samples. As the iridescent spots are typically $0.3 \mathrm{~mm}$ or less in size, the incident beam was focused using an additional $30 \mathrm{~mm}$ focal length lens onto the sample. This produced a cone angle of less than $3^{\circ}$, which can be ignored in reflectance calculations. Light scattered from the sample was collected using a $50 \mathrm{~mm}$ focal length lens masked with a $2.5 \mathrm{~mm}$ (vertical) slit, also giving a cone angle less than $3^{\circ}$. Both incident and scattered beams were passed through linear polarizers to ensure that S-type (polarization perpendicular to the plane of incidence) and P-type (polarization parallel to the plane of incidence) reflections can be measured independently. All reflectance spectra were divided, point by point by the spectrum of the light source, to compensate for the source, monochromator transmission and photomultiplier response. During measurements we fixed the direction of the reflected beam as it is determined by the monochromator, but the direction of the input beam could be changed by mounting the light source on a rotating arm. The sample could also be rotated around a vertical axis. The directions of the various beams and the sample orientation are shown in figure 3.

Ellipsometry was only performed on the bulk shell material and not on the micro-structure. For these measurements the sample was given a high-gloss polish using $\mathrm{Al}_{2} \mathrm{O}_{3}$ powder $(0.05 \mu \mathrm{m}$ grain size $)$ and water. A Gaertner L119 null ellipsometer was used to determine the index of refraction of the shell material in which the micro-structure is embedded. A green HeNe laser at $543 \mathrm{~nm}$ was used as the

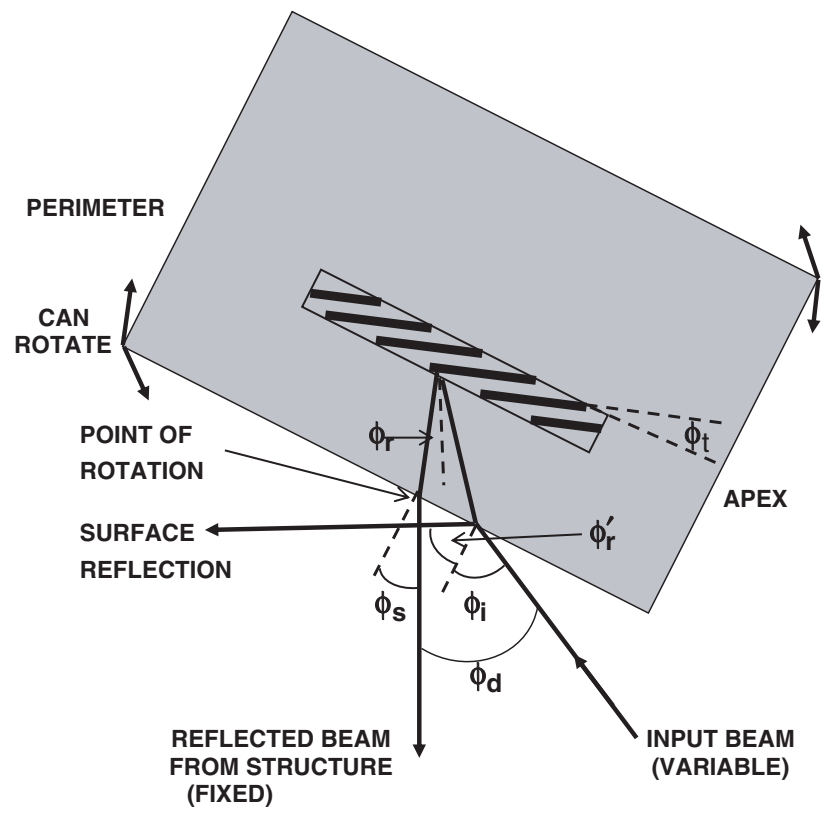

Figure 3. Schematic diagram of a cross section of the shell wall showing the input beam (angle $\phi_{\mathrm{i}}$ ), a beam reflected from the internal thin-film stack (angle $\phi_{\mathrm{r}}$ ) and a beam reflecting from the outer surface (angle $\phi_{\mathrm{r}}^{\prime}=\phi_{\mathrm{i}}$ ). The point of rotation is as close as possible to the intersection of the input and output beams. The direction of the output beam is fixed by the collecting lens and the monochromator, but the direction of the input beam can be changed. The sample can also be rotated in the plane of the page to adjust the internal structure to the correct orientation.

light source as this wavelength is close to the region where the reflectance peaks occur.

Raman spectroscopy was performed with the same monochromator and detector used for the reflectance measurements, using an $\mathrm{Ar}^{+}$laser at $514 \mathrm{~nm}$ and a holographic notch filter to remove stray light at the excitation wavelength.

For scanning electron microscopy (SEM) studies small pieces were embedded in Acryfix (manufactured by Struers) and thin strips were cut, perpendicular to the surface, close to some of the iridescent spots using a diamond cutting wheel. The sides of the strips were then polished with diamond paste to expose the iridescent spots and then washed in alcohol to remove any traces of the polishing residue. Samples were then coated with a thin carbon layer to create a conductive surface as required by the SEM technique. SEM micrographs were then obtained using a Joel 5800 microscope fitted with a Centaurus backscatter electron detector.

\section{Structure of the shell wall}

Figure 1 shows a top view of the mollusc Patella granatine. The rows of rectangular iridescent spots running from the apex to the perimeter are clearly visible. Under low magnification (figure $4(a)$ ) a thin dark grey line (marked A-A) can be seen at about $100 \mu \mathrm{m}$ from the outer surface. This is a stack of alternating dense and less dense material constituting the micro-structure responsible for the iridescence. At a higher magnification (see figure $4(b)$ ) the individual layers in the stack are visible. It is important to note that the individual layers make an angle of roughly $22^{\circ}$ with the envelope of the stack 

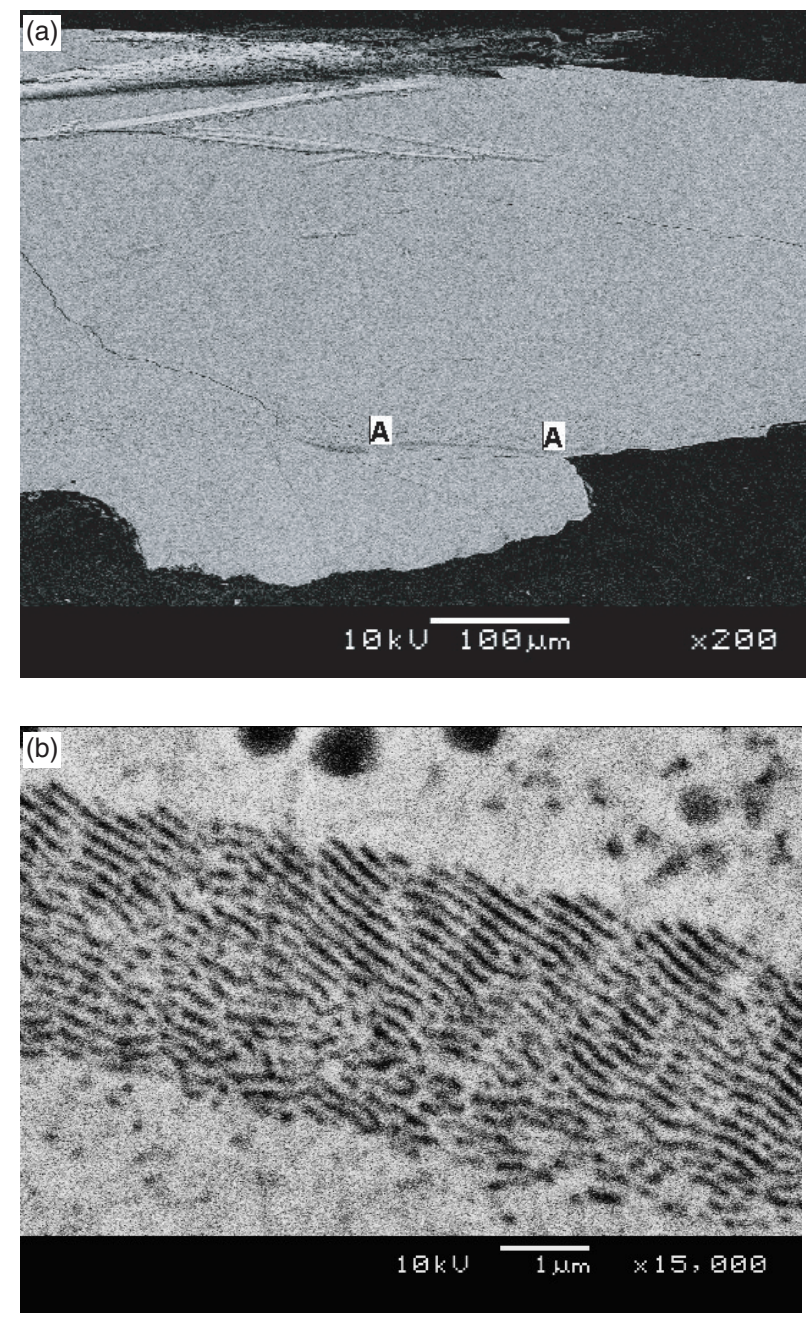

Figure 4. Cross sectional views of the shell wall at low magnification $(a)$ and high magnification $(b)$. In $(a)$ the outer (rough) surface is at the bottom and the inner (smoother) surface is at the top. Note that part of the outer surface broke out while cutting the sample. Normally the thin-film stack, marked as A-A, is completely buried about $100 \mu \mathrm{m}$ from the surface. At high magnification $(b)$ the details of the thin-film stack are visible. Approximately 20 high-density layers (dark) separated by a series of lower-density layers can be seen. The disorder in the lower part of the structure is presumably due to damage during processing.

(angle $\phi_{\mathrm{t}}$ in figure 3), which is approximately parallel to the outer and inner surfaces. Figure 4( $a)$ is a bit misleading as part of the outer surface (lower boundary in figure $4(a)$ ) broke away due to natural erosion and/or processing. Some processing damage is also evident in the lower part of the micro-structure shown in figure $4(b)$. Evidently the resolution of the SEM pictures are insufficient for measuring the exact thickness of the layers in the stack, especially after processing. The optical techniques discussed below provide better quantitative information.

\section{Results and discussion}

\subsection{Reflection and refraction in the shell wall}

Ray tracing involving reflection at the surface, refraction into the shell wall and reflection and interference from the

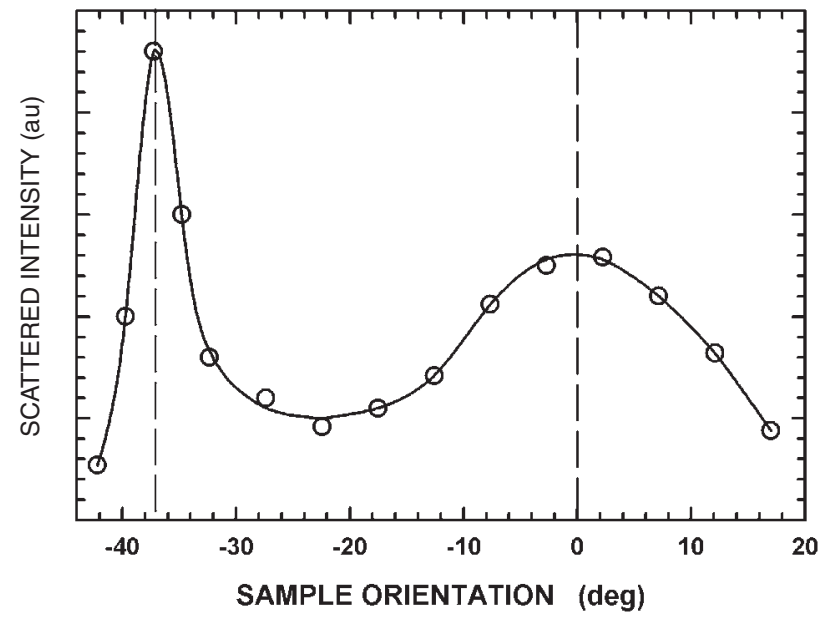

Figure 5. Reflected intensity versus sample orientation for a deviation angle, $\phi_{\mathrm{d}}$, of $20^{\circ}$. The zero position corresponds to a maximum signal from the internal thin-film stack, which produces the relatively broad peak. The narrow peak on the left is caused by a direct reflection from the outer surface.

internal micro-structure plays a crucial role in understanding and interpreting the optical characteristics of the shell. Such a traced path of a typical light ray, relating to the experimental setup in figure 2 , is shown in figure 3 . As pointed out in the experimental section, the direction of the output beam is fixed by the monochromator-detection setup, but the input beam can be varied. It is only limited by the sizes of the focusing and collection lenses to give a minimum value of about $20^{\circ}$ for the deviation angle, $\phi_{\mathrm{d}}$. We started off by setting the input beam at an angle of $20^{\circ}$ to the observation direction $\left(\phi_{\mathrm{d}}=20^{\circ}\right.$ in figure 2). At this stage the exact orientation of the layers in the internal micro-structure is not known, and the only way of getting the internally reflected beam to travel along the preset output direction is to rotate (and tilt) the sample until a maximum signal is observed. A simple visual inspection is used to verify that a direct surface reflection is not responsible for this signal. At this sample position most of the layers in the internal thin-film stack are oriented in such a way that a specular reflection is obtained from them (at an internal angle of reflection $\phi_{\mathrm{r}}$, see figure 3 ) along the preset output direction. A curve of output intensity versus sample position for $\phi_{\mathrm{d}}=20^{\circ}$ is shown in figure 5 , where the broad righthand peak corresponds to reflection (and interference) from the internal layers. The rather large angular width of the peak is mainly due to variations in the orientation of the thin layers constituting the micro-structure.

If the sample is rotated anti-clockwise from this position through a considerable angle, light reflecting directly from the surface will eventually be picked up by the detection setup. This gives the narrow left-hand peak in figure 5 . In this case the width of the peak is mainly due to the angular spread of the input and output beams. Since light is deflected by twice the rotation angle of the sample, the angular difference between the two peaks in figure 5 is $\left(\phi_{\mathrm{i}}+\phi_{\mathrm{s}}\right) / 2$. By definition $\phi_{\mathrm{d}}=\phi_{\mathrm{i}}-\phi_{\mathrm{s}}$, allowing one to calculate the external angles of incidence $\left(\phi_{\mathrm{i}}\right)$ and scattering $\left(\phi_{\mathrm{s}}\right)$ from figure 5 .

In order to perform the thin-film calculations discussed in section 4.4, the internal reflection angle, $\phi_{\mathrm{r}}$, as well as the index of refraction of the bulk shell material, $n_{\mathrm{b}}$, are required. 
Both can be determined from ray tracing if the tilt angle, $\phi_{\mathrm{t}}$, of the internal layers with respect to the surface is known. Unfortunately the value of $n_{\mathrm{b}}$ is extremely sensitive to the choice of $\phi_{\mathrm{t}}$. For example $\phi_{\mathrm{t}}=22^{\circ}$ and $\phi_{\mathrm{d}}=20^{\circ}$ gives $\phi_{\mathrm{i}}=47^{\circ}, \phi_{\mathrm{s}}=27^{\circ}$ and $n_{\mathrm{b}}=1.59$. Keeping everything the same but using $\phi_{\mathrm{t}}=23^{\circ}$ gives $n_{\mathrm{b}}=1.52$. Clearly one cannot determine $\phi_{\mathrm{t}}$ with sufficient accuracy from the SEM results to obtain a useful value for the bulk index of refraction. Fortunately, however, the value of the product, $n_{\mathrm{b}} \sin \phi_{\mathrm{r}}$, is relatively insensitive to $\phi_{\mathrm{t}}$, allowing one to perform some useful calculations (see section 4.4) without knowing the individual values accurately.

Once the correct sample position for a deviation angle $\phi_{\mathrm{d}}=20^{\circ}$ is known, it is a straightforward matter to change the setup for a series of $\phi_{\mathrm{d}}$ values from $20^{\circ}$ to $70^{\circ}$ in steps of $10^{\circ}$. This is done by rotating the sample in $5^{\circ}$ steps (beam direction changes by twice the sample rotation) and by shifting the input beam direction in $10^{\circ}$ steps. As a deviation angle of $70^{\circ}$ corresponds to almost grazing incidence, this was the largest value available for reflectance measurements.

\subsection{Ellipsometry results}

Because of the problem in determining the bulk index of refraction mentioned above, we decided to apply ellipsometry to a small, non-iridescent shell fragment to measure $n_{\mathrm{b}}$ directly.

Ellipsometry measurements were performed by measuring the ellipsometer angles $\psi$ and $\Delta$ experimentally at angles of incidence ranging from $47^{\circ}$ to $72^{\circ}$ in steps of $5^{\circ}$. Values for the unknown optical constants were then varied and $\psi$ and $\Delta$ calculated until a best match (according to a least squares method) was found [7]. The result is shown in figure 6 .

If the index of refraction is taken as real (extinction coefficient $k=0$ ), the calculated $\Delta$ curve is a step-like function, with the step from $180^{\circ}$ to $0^{\circ}$ occurring at the Brewster angle, where $\tan \phi_{\mathrm{i}}=n_{\mathrm{b}}$. The calculated values for $\psi$ lie on two almost straight lines intersecting where the angle of incidence, $\phi_{\mathrm{i}}$, is equal to the Brewster angle [8]. These calculated curves (dashed lines in figure 6) clearly do not fit the experimental data well. Assuming a non-zero value for the extinction coefficient produces an excellent fit for the choice $N_{\mathrm{b}}=n_{\mathrm{b}}-i k=1.55-i(0.03)$ for the complex index of refraction of the bulk material.

As with all ellipsometry measurements the result is entirely dependent on the model assumed for the surface of the sample. A choice of 0.03 for the extinction coefficient implies an absorption coefficient of $\alpha=4 \pi k / \lambda_{\mathrm{o}} \simeq 6900 \mathrm{~cm}^{-1}$, which means that light travelling $100 \mu \mathrm{m}$ to the internal microstructure and back to the surface is attenuated by a factor of $\sim 10^{-60}$ ! Even a $k$-value of 0.01 gives an attenuation factor of $\sim 10^{-20}$, which is obviously impossible. The problem can be resolved by assuming a non-perfect optical surface which changes gradually from $n=1$ to its final value, $n_{\mathrm{b}}$, over a short distance $d$. Using such a 'soft' interface with two variables, $n_{\mathrm{b}}$ and $d$, gives a good fit [7] to the experimental points (solid lines in figure 6) for the choice $n_{\mathrm{b}}=1.55 \pm 0.01$ and $d=15 \mathrm{~nm}$. This is a reasonable choice as the grit size of the $\mathrm{Al}_{2} \mathrm{O}_{3}$ polishing powder is of the order of $50 \mathrm{~nm}$, and with a polishing lap a slightly smoother surface can be achieved than with the grit alone.

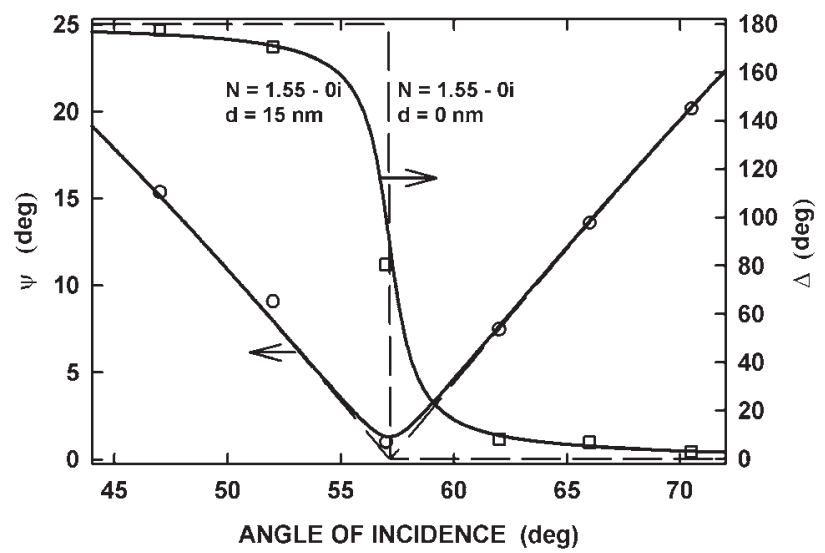

Figure 6. Ellipsometery angles $\psi$ and $\Delta$ as a function of the angle of incidence onto a colourless polished piece of shell. The dashed lines represent a best fit if a non-absorbing perfect interface is assumed, and the solid lines are a best fit when a soft interface of thickness $15 \mathrm{~nm}$ is assumed. For the soft interface we assumed a linear change in refractive index from $n=1.0$ to 1.55 .

\subsection{Raman spectroscopy}

Seashells are normally made from aragonite (a crystalline form of calcium carbonate) in a layered structure, sometimes with organic material between the layers [9]. Aragonite is a birefringent crystal with indices of refraction (sodium D line) 1.531, 1.680 and 1.686 along the principal axes [10]. Assuming a polycrystalline structure, one would therefore expect to observe an average value of around 1.63. This is substantially higher than the value of 1.55 measured by ellipsometry. From this it can be inferred that there are either air gaps or low refractive index organic material between the solid aragonite layers. At this stage insufficient information is available to quantify the structure more closely. We attempted to obtain a qualitative idea of the content of the shell wall using Raman spectroscopy.

Figure 7 shows two Raman spectra obtained from lighter and darker regions of the same shell away from the iridescent patches. The upper spectrum shows two strong peaks at 703 and $1083 \mathrm{~cm}^{-1}$, which are in close agreement with known aragonite peaks at 705 and $1083 \mathrm{~cm}^{-1}[11,12]$. The $703 \mathrm{~cm}^{-1}$ peak distinguishes the material from calcite, which has a Raman peak at $711 \mathrm{~cm}^{-1}$. Two other strong peaks at 1120 and $1510 \mathrm{~cm}^{-1}$ are not present in pure aragonite, but have been observed before on seashells [13] and ascribed to carotenoids having Raman lines at $1107-1118 \mathrm{~cm}^{-1}$ and $1508-1524 \mathrm{~cm}^{-1}$. On the darker patch of the shell the $1083 \mathrm{~cm}^{-1}$ line is just visible and the spectrum is dominated by the carotenoid lines. This confirms the presence of substantial amounts of organic material, which would change the overall refractive index.

\subsection{Reflectance spectra}

Typical reflectance spectra for S- and P-polarized reflections are shown in figure 8 . Both spectra have a full width at half maximum (FWHM) of about $56 \mathrm{~nm}$ and a well defined maximum position. As the deviation angle $\left(\phi_{\mathrm{d}}\right)$ between input and output beams is changed from $20^{\circ}$ (internal reflection angle $\phi_{\mathrm{r}}=5.6^{\circ}$ ) to $70^{\circ}$ (internal reflection angle $\phi_{\mathrm{r}}=16.7^{\circ}$ ) the peak position for S-polarization shifts from 552 to $535 \mathrm{~nm}$. This is 


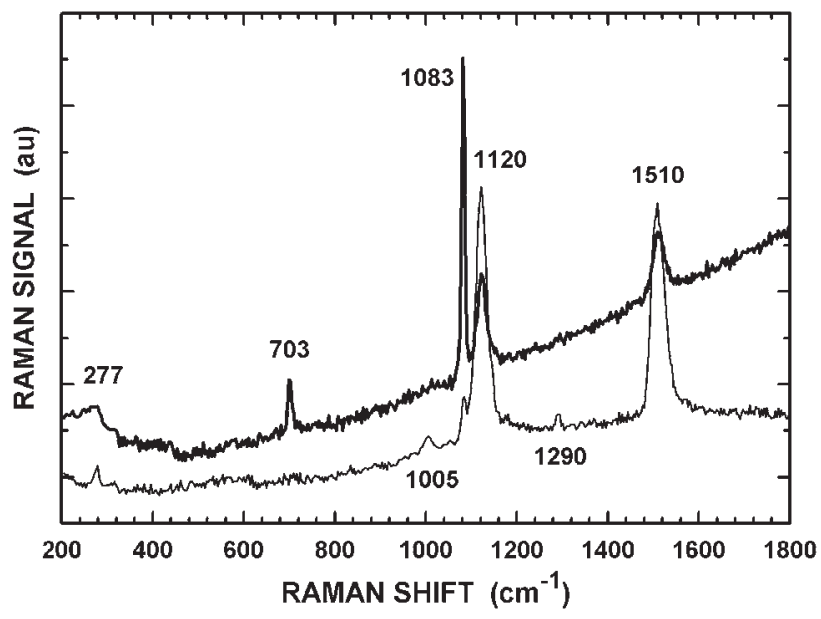

Figure 7. Raman spectra from non-iridescent parts of the shell. The thicker line at the top represents a colourless piece of shell material showing strong aragonite Raman lines at 703 and $1083 \mathrm{~cm}^{-1}$, and the thin line below was obtained from a dark region of the shell where Raman lines closely resembling those of carotenoids dominate the spectrum.

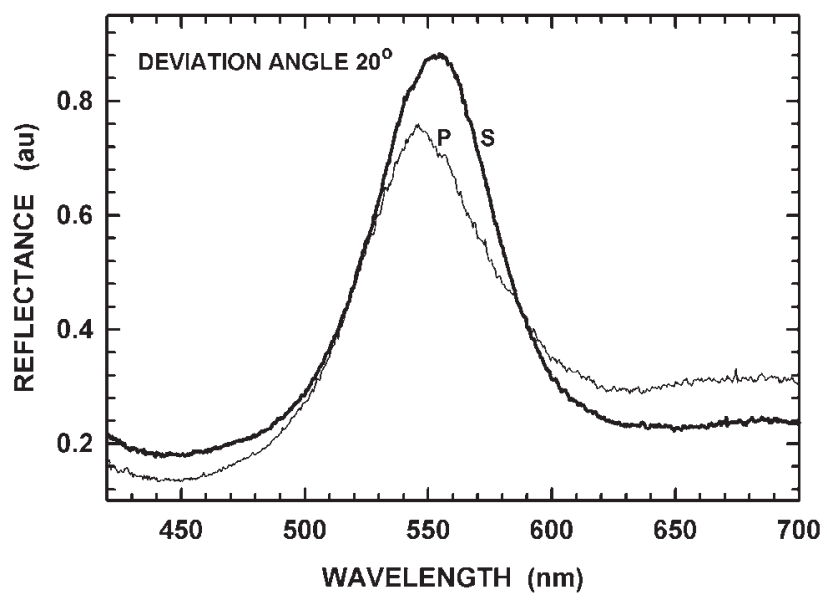

Figure 8. Typical reflectance spectra for S- and P-type reflections at a deviation angle, $\phi_{\mathrm{d}}$, of $20^{\circ}$. Both spectra have an FWHM of about $56 \mathrm{~nm}$. The slight shoulder on the right of the main peaks is presumably due to scattering from the brownish outer surface.

typical for a thin-film stack and allows one to estimate the index of refraction of the dense layers in the stack. Based on the appearance of the SEM micrographs we assumed that the less dense layers have a refractive index close to that of the bulk material of the shell.

For a single high-index $\left(n_{\mathrm{h}}\right)$ layer embedded in a lower index $\left(n_{1}\right)$ medium, interference maxima for reflected light occur when

$$
2 n_{\mathrm{h}} d_{\mathrm{h}} \cos \phi_{\mathrm{h}}=\left(m+\frac{1}{2}\right) \lambda_{\mathrm{o}}
$$

with $d_{\mathrm{h}}$ the layer thickness, $\lambda_{\mathrm{o}}$ the vacuum wavelength and $m$ taking the values $0,1,2$, etc. Using Snell's Law, $n_{\mathrm{b}} \sin \phi_{\mathrm{r}}=$ $n_{\mathrm{h}} \sin \phi_{\mathrm{h}}$, the angles can be converted to the 'incident' angle, $\phi_{\mathrm{r}}$ :

$$
n_{\mathrm{b}}^{2} \sin ^{2} \phi_{\mathrm{r}}=\left(-\frac{1}{16 d_{\mathrm{h}}^{2}}\right) \lambda_{\mathrm{o}}^{2}+n_{\mathrm{h}}^{2}
$$

Hence, from a plot of $n_{\mathrm{b}}^{2} \sin ^{2} \phi_{\mathrm{r}}$ against $\lambda_{\mathrm{o}}^{2}$, independent values of the layer thickness and its index of refraction can be

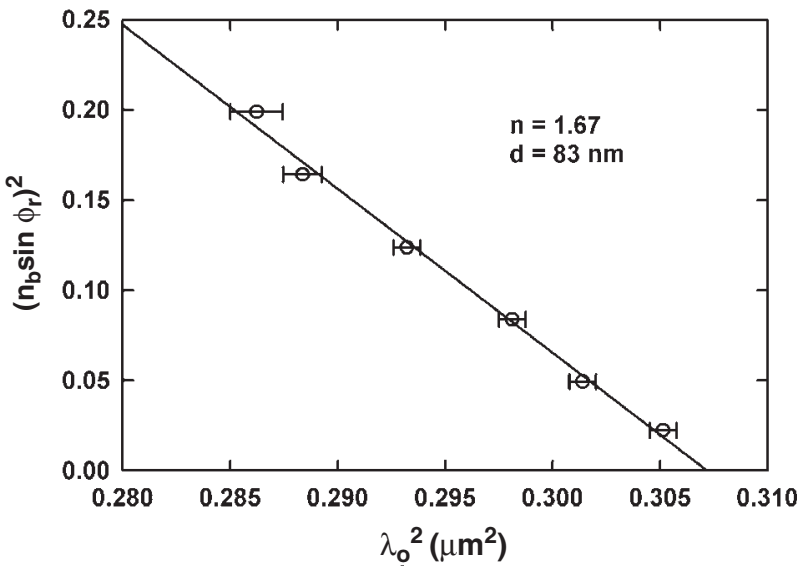

Figure 9. Shift in the reflectance peak due to changes in the internal reflection angle, $\phi_{\mathrm{r}}$. A straight-line fit according to equation (2) yields independent values for the index of refraction as well as the thickness of the high-density layers in the internal thin-film stack.

obtained from the intercept and slope of a straight-line fit. This technique is useful as a parameter fit to the experimental reflectance curves based directly on equation (1) provides a single value for the product $n_{\mathrm{h}} d_{\mathrm{h}}$ (optical layer thickness) and not separate values as can be extracted using equation (2). A quarter wave stack of thin films generates reflection maxima at the same wavelengths, $\lambda_{0}$, as a single layer, and consequently equation (2) is still valid, regardless of the number of layers in the stack. The only effect of adding layers to the stack is that the FWHM of the reflection peak is narrowed and the peak gets higher. A curve according to equation (2) and the corresponding straight-line fit are shown in figure 9. A value of $1.67 \pm 0.03$ for the index of refraction and a thickness of $83 \pm 2 \mathrm{~nm}$ were obtained for the high-density layers. This is quite close to the high refractive index values for crystalline aragonite, suggesting that the high-density layers possess single crystal or partially oriented polycrystalline properties. Randomly oriented crystals can at best give a value equal to the average of the values of the principal axes, i.e. 1.63.

A more detailed analysis of the properties of the thin-film stack can be performed by modelling it according to the well known matrix method [14]. The results of such an analysis are shown in figure 10. Under the conditions inside the shell wall at a reflection angle $\phi_{\mathrm{r}}=5.5^{\circ}\left(\phi_{\mathrm{r}}=20^{\circ}\right)$, a single layer gives a reflectance of only $0.56 \%$ and a FWHM of as much as $734 \mathrm{~nm}$ (from 368 to $1102 \mathrm{~nm}$ ). Adding more layers rapidly decreases the FWHM and increases the reflectance. According to the model the experimentally observed FWHM of $56 \mathrm{~nm}$ should be reached for 11 (perfect) high-density layers in the stack. From figure $4(b)$ it can be seen that there are actually close to 20 high-density layers present, but as they are clearly far from optically perfect, they perform as an equivalent stack of 11 high-density layers. If all 20 layers fully participated in the reflection/interference process, the FWHM would have been in the region of $40 \mathrm{~nm}$. Also shown in figure 10 is the reflectance versus number of high-density layers. Assuming that the actual micro-structure acts like an 11-layer structure, one can expect a reflectance of the order of $46 \%$, which is certainly much better than the $0.56 \%$ of a single layer. Absolute reflectance measurements could not be performed due to excessive scattering from the outer surface. 


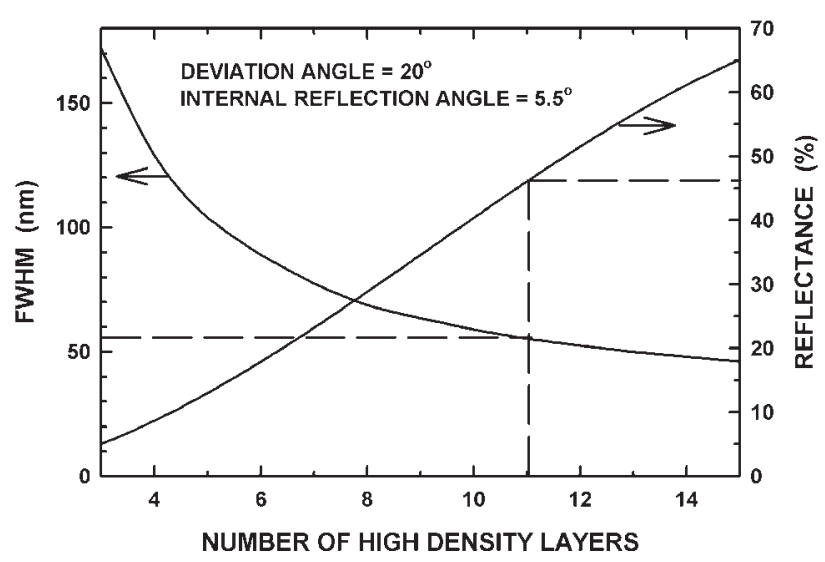

Figure 10. Calculated curves showing the peak reflectance as well as the FWHM of the peaks versus the number of high-density layers in a quarter wave stack of $n=1.67$ films embedded in an $n=1.55$ medium. The dashed lines indicate that the experimentally observed FWHM $(56 \mathrm{~nm})$ is reached with 11 optically perfect layers. Such a thin-film stack produces a peak reflectance of $46 \%$.

It is interesting to note that the shell investigated earlier [6] exhibited a much broader FWHM of around $90 \mathrm{~nm}$. In spite of the much smoother external surface of Helcion pruinosus it seems that the internal surfaces are somewhat rougher than those in Patella granatina.

As for Helcion pruinosus, the reflection maximum for P-polarization is blue-shifted by about $8.5 \mathrm{~nm}$ (for $\phi_{\mathrm{d}}=20^{\circ}$ ). This could be due to a reduced optical thickness, i.e. a slightly lower index of refraction, of the layers for this polarization. According to the matrix model a change in refractive index to 1.62 for the high index layers and no change for the low index layers will shift the reflection peak by this amount. As the optical thickness of the low index layers are now no longer a quarter wavelength and the contrast in the index of refraction between layers is reduced, the reflection drops to around $20 \%$ and the FWHM increases slightly to $47 \mathrm{~nm}$. Once again this is an indication of single crystal properties in the high-density layers.

\section{Conclusion}

We have shown that the iridescent green spots on Patella granatina are caused by a buried quarter wave thin-film stack at about $100 \mu \mathrm{m}$ below the outer surface of the shell. This micro-structure is tilted by an angle of roughly $22^{\circ}$ with respect to the outer surface, allowing the iridescence to be observed when viewing the shell at close to grazing incidence from the apex to the perimeter. The micro-structure is about twice as deep below the surface as for Helcion pruinosus, but it seems to be of a higher optical quality, as the spectral width of the reflection peak is somewhat narrower. In spite of the fact that the two shells belong to different genera and species, the layer thickness and index of refraction are very similar. In both cases the high-index layers seem to be made from fairly pure crystalline aragonite.

Ellipsometry was used to determine the index of refraction of the bulk material, and Raman spectroscopy was used to confirm that the bulk material is not pure aragonite, but contains a substantial amount of organic material.

\section{References}

[1] Durrer H and Villiger W 1970 Z. Zellforsch 109 407-13

[2] Ghiradella H 1991 Appl. Opt. 30 3492-500

[3] Brink D J and Lee M E 1998 Appl. Opt. 37 4213-7

[4] Brink D J and Lee M E 1999 Appl. Opt. 38 5282-9

[5] Brink D J and van der Berg N G 2004 J. Phys D: Appl. Phys. 5 813-8

[6] Brink D J and van der Berg N G 2002 Appl. Opt. 41 717-22

[7] Jellison G E 1998 Thin Solid Films 234 416-22

[8] Azzam R M A and Bashara N M 1989 Ellipsometry and Polarized Light (Amsterdam: North Holland) pp 277-8

[9] Weiner S and Addadi L 1997 J. Mater. Chem. 7 689-702

[10] Winchell A N and Winchell W 1967 Elements of Optical Mineralogy (New York: Wiley) pp 117-8

[11] Dickinson S R and McGrath K M 2001 Analyst 126 1118-21

[12] Gauldie R W, Sharma S K and Volk E 1997 Comp. Biochem. Physiol. 118A 753-7

[13] Withnall R, Chowdry B Z, Silver J, Edwards H G M and Oliveira L F C 2003 Spectrochim. Acta A59 2207-12

[14] Guenter R D 1990 Modern Optics (New York: Wiley) pp 124-8 